\title{
Editorials
}

\section{Antiganglioside antibodies in peripheral neuropathies}

Over the last decade or so serum antibodies have been shown to be associated with three groups of neurological disorders: antibodies to ion channels and other functional molecules in myasthenia gravis and related disorders; antibodies to cytoplasmic CNS antigens in paraneoplastic disorders; and antibodies to peripheral nerve antigens. Whereas the first two groups have well established diagnostic or prognostic uses, ${ }^{1}$ the relevance of antibodies to peripheral nerve antigens, particularly gangliosides, is less clear.

Gangliosides are sialic acid containing glycosphingolipids that are highly concentrated in certain parts of the nervous system, and which can be obtained commercially. Many laboratories are now performing in-house enzyme linked immunosorbent assays (ELISA) for antibodies to GM1, and to a lesser extent GQ1b (for nomenclature see Willison ${ }^{2}$ ), and one needs to consider whether these antibodies are specific for disease, represent part of a pathogenic immune response against neuronal antigens, and whether their detection is helpful in the management of the patient.

Antiganglioside antibodies are typically found in inflammatory peripheral neuropathies. Miller Fisher syndrome is an acute, monophasic illness associated with ophthalmoplegia, ataxia, and areflexia. IgG antibodies to ganglioside GQ1b are present in $>90 \%$ of patients, and in some patients with related syndromes, ${ }^{2}$ at the peak of the disease, but they usually decrease to undetectable levels within a few weeks. Detection of anti-GQ1b antibodies confirms the diagnosis, although the patient has often improved before the result is available. IgM antibodies to GQ1b and other disialylated gangliosides, sometimes with associated cold agglutinin activity, are found in a very rare chronic ataxic neuropathy, with symptoms similar to those in Miller Fisher syndrome. ${ }^{2}$ On the other hand, IgG, IgA, and IgM serum antibodies to GM1 and other Gal ( $\beta 1-3)$ GalNAc bearing gangliosides are found in the GuillainBarré syndrome which is usually associated with inflammatory infiltrates and demyelination of motor and sensory peripheral nerves, but can also include axonal damage. In addition, IgM GM1 antibodies are found in up to $50 \%$ of patients with multifocal motor neuropathy, a rare chronic disorder with asymmetrical muscle weakness associated with demyelination and focal block of nerve conduction.

Several investigators have claimed to detect antibodies to GM1 in patients with other neurological or immunological conditions, giving the antiganglioside antibodies a reputation for promiscuity that is probably ill deserved. Using standardised techniques, and controlling for absorption of immunoglobulins to the uncoated ELISA plates, antiganglioside antibodies are only present at low titre (for example, $<1: 400$ ) in healthy or other disease controls $^{2}$ and can reach titres of $>1: 6400$ or greater in Guillain-Barré syndrome or multifocal motor neuropathy. Positive titres of anti-GQ1b antibodies are very infrequent in controls, and values $>1: 50$ are generally considered positive.
There is convincing evidence for a pathogenic action of serum antibodies in anti-GQ1b antibody associated conditions. Sera or IgG preparations from patients with Miller Fisher syndrome showed direct effects on the function of the motor nerve terminal in a mouse preparation, although the mechanism of action and the role of complement both need to be clarified. ${ }^{2}{ }^{3}$ In addition, a cloned monoclonal antibody from a patient with chronic ataxic neuropathy bound to the motor nerve terminal in a passive immunisation model and reduced neurotransmitter release. ${ }^{4}$ Thus these conditions, which benefit from plasma exchange, appear to be mediated at least in part by serum antibodies. With respect to anti-GM1 antibodies the situation is less clear. GM1 ganglioside is highly concentrated at the nodes of Ranvier, with similar localisation to that of the sodium channels that propagate the nerve impulse. Thus it was reasonable to assume that antibodies to GM1 might affect nerve conduction by altering sodium channel function, or lead to complement mediated damage of the nodal membrane. Several reports indicated that antibodies to GM1 or related gangliosides can directly alter nerve conduction after exposure of intact nerve preparations in vitro or after intraneural injection, but others have consistently failed to demonstrate any effect (reviewed by Hartung et $a l^{3}$ ). Nevertheless, these patients also benefit from plasma exchange and intravenous immunoglobulin, implicating serum factors.

Both Guillain-Barré syndrome and Miller Fisher syndrome often present after a viral or bacterial infection. Over the last few years, there have been many reports of cases associated with particular strains of Campylobacter jejuni, and lipopolysaccharide preparations from certain strains of $C$ jejuni express ganglioside-like epitopes. ${ }^{23}$ Guillain-Barré syndrome occurs as an epidemic in certain parts of rural China; the incidence of $C$ jejuni infection is particularly high in cases with an acute motor axonal form. ${ }^{5}$ Although many Chinese patients had antibodies to GM1, however, overall these did not correlate with antibodies to $C$ jejuni or with the pattern of disease. Similarly, of 96 consecutive cases of Guillain-Barré syndrome or Miller Fisher syndrome studied by Rees et al in the United Kingdom, ${ }^{6} 26 \%$ had evidence of $C$ jejuni infection preceded by a diarrhoeal illness. These cases had more severe motor symptoms, often with acute axonal damage, and a less good prognosis. Although in this study anti-GM1 antibodies were significantly more common in $C$ jejuni positive than in $C$ jejuni negative patients, infection was a more important prognostic indicator than anti-GM1 antibody positivity.

Thus there is an emerging story that antiganglioside antibodies can result from cross reactivity with bacterial antigens, and that some of them at least can have direct-probably complement mediated-effects on peripheral nerve function. Nevertheless, there is much that we need to know about the relation of these antibodies with $C$ jejuni and other preceding infections, and about the relative roles of antibodies (and possibly 
other inflammation related serum factors) in the pathogenesis of the peripheral nerve disorders.

ANGELA VINCENT

Department of Clinical Neurology, University of Oxford, Institute of Molecular Medicine, fohn Radcliffe Hospital, Headington, Oxford OX3 9DS, UK

1 Lang B, Vincent A. Autoimmunity to ion-channels and other proteins in paraneoplastic disorders. Curr Opin Immunol 1996;8:865-71.

2 Willison HJ. Ganglioside antibodies. In: Peter JB, Shoenfeld Y. Autoantibodies. Amsterdam: Elsevier Science, 1996:277-84.
3 Hartung H-P, Toyka KV, Griffin JW. Guillain-Barré syndrome and chronic inflammatory demyelinating polyradiculoneuropathy. In: Antel J, Birnbaum G, Hartung H-P, eds. Clinical neuroimmunology. Oxford: Blackwell, 1998:294-306.

4 Willison HJ, O'Hanlon GM, Paterson G, et al. A somatically mutated human anti-ganglioside IgM antibody that induces experimental neuropathy in mice is encoded by the variable region heavy chain gene, V1-18. f Clin Invest 1996; 97:1155-64.

5 Ho TW, Mishu B, Li CY, et al. Guillain-Barré syndrome in northern China. Relationship to Campylobacter jejuni infection and anti-glycolipid antibodies. Brain 1995;118:597-605.

6 Rees JH, Gregson NA, Hughes RA. Anti-ganglioside GM1 antibodies in Guillain Barré syndrome and their relationship to Campylobacter jejuni infection. Ann Neurol 1995;39:809-16.

\section{How much work do you do in a day?}

Does this question induces feelings of pride, guilt, satisfaction, or indignation? Whatever the response, most histopathologists will find it difficult to answer it in a meaningful, quantified, reproducible way. It is a question which often needs to be answered, particularly when manpower changes or changes in working practices are contemplated.

Historically, the diagnostic workload of histopathologists has been assessed by the number of specimens reported. It is in this way that the Royal College of Pathologists frames its staffing recommendations. The approach is crude. Not all specimens are alike; some take much more work than others. In the past this may not have mattered, as each pathologist shared a similar pattern of work; but subspecialisation is advancing and will increasingly make "specimen counting" a misleading exercise. Furthermore, the workload per specimen is changing. The average number of "items of information" in a single histopathology report probably bears some relation to the work involved in its generation; this measure was found to have increased by over $200 \%$ between 1940 and $1990 .{ }^{1}$ The trend has almost certainly continued, and with the development of minimum datasets it seems likely to accelerate.

There have been several attempts to develop more sensitive methods of measuring whole laboratory workloads, of which WELCAN units are perhaps best known in the United Kingdom. Even in assessing laboratory costs the system has some problems, ${ }^{2-4}$ but it simply was not designed to measure the workload of histopathologists.

The extreme solution to this problem would perhaps be a large time and motion study to assess just how long an average pathologist takes to produce a report on each type of specimen. Workloads could then be calculated by multiplying each specimen reported by its measured weighting and adding the results together. But who would volunteer to run such a study? How would it be kept up to date? Would it be worth the effort?

In this context, the paper by Suvarna and Kay in the July issue $^{5}$ represents a compromise. By discussion and consensus, they have sought to develop a system in which different specimens are assigned different weights in calculating a pathologist's workload. Advantages over other systems are demonstrated. Although superficially more complex than simple specimen counting, most modern laboratory computer systems should be able to automate this sort of process with no more than minor modifications to the software. The hard work is not in using such a system, but in developing and validating it. Suvarna and Kay have made a start, but they acknowledge that their weightings are unlikely to be acceptable in all laboratories and they invite assistance. The relation between a "unit" and a measure of pathologist's time remains a little vague, and there is no obvious mechanism to update the system as new developments require additional or alternative approaches. If this is to work, what is needed next is for other laboratories to carry out similar weighting assessments, with a view to developing a national consensus. Further validation by direct observation of pathologists' working practices will be needed, in a variety of laboratories.

A sophisticated, validated method could have benefits beyond those proposed by Suvarna and Kay. For example, I may suspect that I spend too long examining endometrial biopsies, but tire too quickly when examining those innumerable prostatic chips. I find that I am working long hours; is this because I am obsessional with the high power objective, or am I being asked to do more work than is reasonable? How can I know? Truth is elusive, but with a national consensus on current practice we would at least have a benchmark for comparison. Most pathologists would welcome this as a form of self assessment, but there may be concern that self assessment could be replaced by peer or managerial assessment. Even so, is it not preferable to be assessed on the basis of meaningful measurements rather than specimen counts? Perhaps not-if the inadequacy of the data is a useful excuse for its dismissal.

Do we need all this? Is it worth it? Do doctors in other specialties go to this sort of trouble? One might think that private medicine might provide a test of motivation, as histopathologists are paid not a salary, but by item of work. Here surely is an incentive to measure work accurately; but instead we usually see old, crude systems of workload measurement. Does this mean that there is no appetite for more accuracy?

Specimen counting may have been sufficient in the past, and there may be fear of the controversies that will be provoked by any change; but with increasing demands on pathologists' time, with subspecialisation making specimen counting meaningless, and with laboratory computerisation making it easier to be more accurate, surely our methods of workload measurement will have to improve.

P N FURNESS

Department of Pathology, Leicester General Hospital, Gwendolen Road, Leicester LE5 4PW, UK; email: pnf1@le.ac.uk

1 Cross SS, Bull AD. Is the informational content of histopathological reports increasing? f Clin Pathol 1992;45:179-80.

2 Bennett CH. WELCAN UK: its development and future [editorial]. $\mathcal{F ~ C l i n}$ Pathol 1991;44:617-20.

3 Sims J. Welcan units [editorial]. Cytopathology 1992;3:201-2.

4 Tarbit IF. Laboratory costing system based on number and type of test: its association with the Welcan workload measurement system. $\mathcal{F}$ Clin Pathol 1990;43:92-7.

5 Suvarna SK, Kay MS. KU activity: a method for measuring histopathologists' workloads. F Clin Pathol 1998;51:530-534. 